Marquette University

e-Publications@Marquette

$1-1-2016$

\title{
Does Exercise Decrease Pain via Conditioned Pain Modulation in Adolescents?
}

Stacy Stolzman

Marquette University

Marie K. Hoeger Bement

Marquette University, mariehoeger.bement@marquette.edu

Accepted version. Pediatric Physical Therapy, Vol. 28, No. 4 (Winter 2016): 470-473. DOI. (C) 2016 Academy of Pediatric Physical Therapy of the American Physical Therapy Association. Used with permission. 


\title{
Does Exercise Decrease Pain via Conditioned Pain Modulation in Adolescents?
}

\author{
Stacy Stolzman \\ Health and Human Performance Department, Exercise Physiology Program, \\ Concordia University Wisconsin, Mequon, WI \\ Marie Hoeger Bement \\ Department of Physical Therapy, Marquette University, Milwaukee, WI
}

\begin{abstract}
Purpose: Pain relief after exercise, exercise-induced hypoalgesia (EIH), is established across the lifespan. Conditioned pain modulation (CPM: pain inhibits pain) may be a mechanism for EIH.

Methods: In 55 adolescents, pressure pain thresholds were measured before and after exercise (deltoid, quadriceps, and nail bed) and during CPM at the nail bed and deltoid test stimulus sites. The relationship between EIH and CPM was explored.

Results: EIH occurred at deltoid and quadriceps; CPM occurred at nail bed and deltoid. CPM and EIH correlated at deltoid; adolescents with greater CPM experienced greater pain relief after exercise. At this site, CPM predicted 5.4\% of EIH. Arm lean mass did not add a significant effect. Peak exercise pain did not influence EIH. Adolescents with none, minimal, moderate, or severe peak exercise pain experienced similar EIH.

Conclusions: A potential relationship exists between CPM and EIH in adolescents. Pediatric physical therapists should consider the CPM response when prescribing exercise as a pain management tool.
\end{abstract}

Pediatric Physical Therapy, Vol. 28, No. 4 (Winter 2016): pg. 470-473. DOI. This article is ( Lippincott Williams \& Wilkins, Inc. and permission has been granted for this version to appear in e-Publications@Marquette. Lippincott Williams \& Wilkins, Inc. does not grant permission for this article to be further copied/distributed or hosted elsewhere without the express permission from Lippincott Williams \& Wilkins, Inc.. 


\section{Introduction}

A decrease in pain after exercise is exercise-induced hypoalgesia (EIH), which is well established in adults $\frac{1-3}{-3}$ and more recently in adolescents across the weight status. $\underline{4}$ EIH is an example of endogenous pain modulation and produces systemic effects; pain relief occurs throughout the body. $\underline{2}$ In young healthy adults, aerobic exercise of higher intensity produces greater EIH compared with lower intensity exercise, $\underline{5}$ and isometric contractions that are reported as painful produce greater EIH compared with nonpainful contractions. $\underline{6}$

Conditioned pain modulation (CPM) is the concept that "pain inhibits pain" and is a measure of central endogenous pain modulation. $\underline{7}$ In the clinic, any physical therapy intervention that is reported as unpleasant (ie, exercise, thermal modalities, and electrical stimulation) could work through the mechanism of CPM. With CPM in the research setting, a noxious stimulus (conditioning stimulus) decreases pain perception of a subsequent noxious stimulus (test stimulus). $\frac{8}{}$ A greater conditioning stimulus produces greater CPM. $\frac{8}{}$ Young healthy adults and adolescents demonstrate a consistent robust CPM response that tends to decline with increasing age. $\frac{9-14}{14}$ Taken together, these results suggest exercise that is painful may activate descending inhibitory pathways, resulting in subsequent pain relief (ie, EIH). In young and older adults, CPM predicts EIH. $\underline{31}, \underline{15}$ Although not causal, this suggests that CPM may produce an additive EIH effect when exercise is reported as painful.

Previously, we have shown that EIH and CPM exist in adolescents and are individually correlated with lean mass regardless of weight status. $\underline{4}, \underline{13}$ The purpose of this study is to determine whether a relationship exists between CPM and EIH in these adolescents. We hypothesize that the CPM and EIH response in adolescents are related and potentially linked through lean mass.

\section{Methods}

\section{Subjects}

Sixty-two adolescents (15.1 \pm 1.8 years; 29 male and 33 female) were recruited from Milwaukee, Wisconsin. These participants were enrolled as part of a larger research study investigating the association between inflammatory markers, physical fitness, and pain in adolescents of varying weight status.

Pediatric Physical Therapy, Vol. 28, No. 4 (Winter 2016): pg. 470-473. DOI. This article is ( Lippincott Williams \& Wilkins, Inc. and permission has been granted for this version to appear in e-Publications@Marquette. Lippincott Williams \& Wilkins, Inc. does not grant permission for this article to be further copied/distributed or hosted elsewhere without the express permission from Lippincott Williams \& Wilkins, Inc.. 
Adolescents participated in 3 experimental sessions. The first session included measurement of weight status and experimental pain (pressure pain threshold [PPT]) to familiarize the adolescents to the pressure algometer (Algomed). 4 After the first session, adolescents participated in either the EIH or CPM session in a counterbalanced manner. The EIH session involved measurement of PPTs 3 times at 3 sites-left nail bed, left deltoid muscle, and right quadriceps muscle-before and after a maximal aerobic treadmill test (V02Max Bruce Protocol). 4 The magnitude of EIH is the increase in PPTs after exercise. 4 The adolescents rated their exercise pain using a Numerical Rating Scale (NRS) 0 to 10 with the anchors: 0 as "no pain" and 10 as "worst pain" during each stage of the treadmill test and upon completion. The CPM session involved body composition testing (dual-energy x-ray absorptiometry [DXA] scan) and the CPM protocol. $\underline{12} \underline{13}$ For the CPM protocol, PPTs were measured at the nail bed and deltoid muscle with the right foot in a control condition (room temperature cool water bath) followed by a noxious condition (ice water bath); the time between the neutral and ice water conditions was 20 minutes. $12 \underline{13}$ The ice water was the conditioning stimulus and PPTs were the test stimulus. Two trials at each site (nail bed and deltoid) were completed for CPM to limit the exposure to the ice water condition. The absolute difference in PPTs between the noxious and control conditions is the magnitude of CPM. $\underline{11} \underline{13}$

\section{Statistical Analysis}

The 2 PPTs were averaged for the CPM testing at each site (CPMNail and CPMDelt);

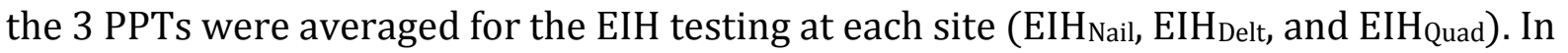
addition, the EIH and CPM were averaged across all sites (EIH $\left.\mathrm{Ell}_{\text {and }} \mathrm{CPM}_{\mathrm{All}}\right)$. For each EIH and CPM session, repeated-measures analyses of variance (ANOVAs) (trial [pre- and postexercise] or [cool water and ice water]) were done with site as a factor within the analysis. Post-hoc Pearson correlations were computed for EIH at the deltoid muscle (EIHDelt), quadriceps muscle (EIHQuad), and average EIH across muscles (EIHDeltQuad) with CPM at the nail bed (CPMNail), deltoid muscle (CPMDelt), and average CPM at the 2 sites (CPM $\left.M_{\text {NailDelt}}\right)$. From significant Pearson correlations, a regression analysis was completed with EIHDelt as the dependent variable and CPMDelt entered as step 1. Because lean mass has been shown to influence EIH and CPM, $\underline{4} \underline{13}$ lean mass of the left arm taken from the DXA results was included in step 2. Data were analyzed using Statistical Package for the Social Sciences (SPSS, version 23, IBM, Chicago, Illinois) for statistics.

To determine whether pain during exercise contributed to the EIH response, adolescents were classified into groups based on the peak pain ratings (NRS 0-10) during the treadmill test: no pain (0/10 NRS), minimal pain (1-3/10 NRS), moderate pain (46/10 NRS), and severe pain (7-10/10 NRS). 16,17 Repeated-measures ANOVA (trial [pre and

Pediatric Physical Therapy, Vol. 28, No. 4 (Winter 2016): pg. 470-473. DOI. This article is ( Lippincott Williams \& Wilkins, Inc. and permission has been granted for this version to appear in e-Publications@Marquette. Lippincott Williams \& Wilkins, Inc. does not grant permission for this article to be further copied/distributed or hosted elsewhere without the express permission from Lippincott Williams \& Wilkins, Inc.. 
postexercise] $\times$ site [deltoid and quadriceps muscles]) was done with peak exercise pain groups (no pain, minimal pain, moderate pain, and severe pain) $\underline{16} \underline{17}$ as a between-subject factor. An [alpha] level of $\mathrm{P}<.05$ was used for all analyses.

\section{Results}

Fifty-five adolescents completed the EIH and CPM protocols (Table 1). Detailed EIH and CPM results are reported elsewhere, $\underline{4} \underline{13}$ but in summary, fit and unfit adolescents reported an increase in PPTs after exercise (EIH) and were unchanged with quiet rest. 4 For CPM, PPTs increased in the ice water compared with the neutral water for adolescents in a similar manner across weight status (normal vs overweight/obese) and $\operatorname{sex} . \underline{13}$

Within the exercise session alone, EIH is site specific (trial $\times$ site: $\mathrm{P}<.001$ ) with significant increases in pain thresholds at the deltoid $(\mathrm{P}=.004)$ and quadriceps $(\mathrm{P}$ $<.001)$ but no significance at nail bed $(\mathrm{P}>.05)$. Within the CPM session alone, CPM is similar across sites (trial $\times$ site: $\mathrm{P}=.47$ ) with significant increase in pain threshold at the nail bed $(\mathrm{P}<.001)$ and deltoid $(\mathrm{P}<.001)$ whereas the foot is submerged in ice water compared with neutral water.

CPMDelt was positively correlated with EIHDelt $(r=0.27, \mathrm{P}=.05)$ in that adolescents who experienced greater conditioned pain modulation at the deltoid muscle also experienced greater pain relief after exercise at the deltoid muscle (Table 2 and Figure 1). $\mathrm{CPM}_{\text {Delt }}$ predicted $5.4 \%$ of the variance in the EIHDelt response $(\mathrm{F}=4.074, \mathrm{P}=.049)$ and lean mass did not add a significant effect $(\mathrm{F}=2.09, \mathrm{P}=.13)$.

\section{Peak Pain With Maximal Aerobic Exercise}

EIH was similar between the pain groups (trial $\times$ site $\times$ peak pain: $\mathrm{P}>.05$ ); peak pain reported during maximal aerobic exercise did not influence EIH (Figure 2).

\section{Discussion}

This is the first study to support that CPM predicts EIH in adolescents after maximal treadmill running. We have previously shown this predictive relationship in adults after maximal isometric contractions held to task failure.11 In young and older adults, CPM uniquely predicted $8.8 \%$ of the variance in the EIH.11 Taken together, there is a predictive relationship between CPM and EIH that occurs from adolescence through older adulthood and after exhaustive aerobic and isometric exercise. Others have shown a positive relationship between CPM and EIH in adults after 15 minutes of cycling at moderate/high intensity. .15 Conversely, there was no association between CPM and EIH after low- and high-intensity cycling or isometric contractions. $\underline{3}$ Thus, there are equivocal results in the

Pediatric Physical Therapy, Vol. 28, No. 4 (Winter 2016): pg. 470-473. DOI. This article is ( Lippincott Williams \& Wilkins, Inc. and permission has been granted for this version to appear in e-Publications@Marquette. Lippincott Williams \& Wilkins, Inc. does not grant permission for this article to be further copied/distributed or hosted elsewhere without the express permission from Lippincott Williams \& Wilkins, Inc.. 
relationship between EIH and CPM, which may be related to exercise dose with more consistent results when the exercise is completed to exhaustion.

The mixed results may also be related to the measurement site. Our study demonstrated that the CPM and EIH relationship was site specific. There was a positive association between the CPM response at the deltoid muscle and the EIH response at the deltoid muscle only. When comparing CPM and EIH averages across testing sites or between dissimilar sites (eg, comparing quadriceps muscle with deltoid muscle), there were no associations. Similarly, Lemley et al $\underline{11}$ reported that CPM was predictive of EIH when the index finger was the measurement site for both protocols. One potential explanation is that the sensitivity to change for both CPM and EIH is similar when using the same site for both protocols. Similar to our protocol, Vaegter et al $\underline{3} \underline{15}$ used multiple sites (leg and arm) in the measurement of EIH and CPM with mixed results; although the $\mathrm{CPM}$ and EIH response by site was not measured but rather the average CPM and EIH across sites. Our results in which the systemic responses of CPM and EIH were measured at the same measurement site (deltoid) show a significant small effect.

No studies to our knowledge have investigated the role of body composition in the relationship between EIH and CPM. Independent of exercise, Price et al 18 has shown no difference in CPM efficiency between obese and normal-weight adults using a test site with little excess subcutaneous fat (forehead). Previously, we have shown in adolescents that lean mass was related to both the CPM and EIH responses; lean mass of the arm uniquely predicted $10 \%$ of the CPM magnitude and lean mass of the body was correlated with the EIH magnitude. $\underline{43}$ Despite previous research showing that lean mass was related to both CPM and EIH independently, lean mass does not appear to account for the relationship between CPM and EIH.

Because exercise is often painful, peak pain during exercise was measured as a potential conditioning stimulus. The average peak pain reported during maximal aerobic exercise by the adolescents was moderately painful with a wide range of peak pain reported. Furthermore, peak pain reported during exercise did not influence the EIH response in our adolescent population. Thus, despite the relationship between CPM and $\mathrm{EIH}$ at the deltoid muscle, our results show that exercise pain does not influence the EIH response; adolescents who experience none, minimal, moderate, or severe peak pain during exercise experience similar EIH. When physical therapists use exercise for pain relief in an adolescent population, it is not necessary for the exercise to be painful in order for pain relief to occur.

Considering the small relationship between EIH and CPM and the similar EIH across the peak pain exercise groups, our results suggest that there are likely multiple

Pediatric Physical Therapy, Vol. 28, No. 4 (Winter 2016): pg. 470-473. DOI. This article is @ Lippincott Williams \& Wilkins, Inc. and permission has been granted for this version to appear in e-Publications@Marquette. Lippincott Williams \& Wilkins, Inc. does not grant permission for this article to be further copied/distributed or hosted elsewhere without the express permission from Lippincott Williams \& Wilkins, Inc.. 
mechanisms that are responsible for EIH. $\underline{11} \underline{19}$ For example, we have previously shown the magnitude of EIH is related to sedentary behavior in adolescents across weight status. $\underline{4}$ Future pediatric research is necessary to evaluate other multifactorial mechanisms such as psychosocial factors as well as specific pain conditions in a variety of ages. From a clinical perspective, assessment of CPM in pediatric populations by physical therapists has the potential to assist with clinical decision making about the use of exercise as a pain management tool in adolescents. With the current focus on decreasing pain medications, alternate pain relief options, such as exercise and endogenous pain modulation, are necessary for adolescents experiencing pain.

\section{References}

${ }^{1}$ Koltyn KF. Exercise-induced hypoalgesia and intensity of exercise. Sports Med. 2002;32(8):477-487.

${ }^{2}$ Kosek E, Lundberg L. Segmental and plurisegmental modulation of pressure pain thresholds during static muscle contractions in healthy individuals. Eur J Pain. 2003;7(3):251-258.

3Vaegter HB, Handberg G, Graven-Nielsen T. Similarities between exercise-induced hypoalgesia and conditioned pain modulation in humans. Pain. 2014;155(1):158-167.

${ }^{4}$ Stolzman S, Danduran M, Hunter SK, Bement MH. Pain response after maximal aerobic exercise in adolescents across weight status. Med Sci Sports Exerc. 2015;47(11):2431-2440.

${ }_{5}$ Naugle KM, Fillingim RB, Riley JL 3rd. A meta-analytic review of the hypoalgesic effects of exercise. J Pain. 2012;13(12):1139-1150.

${ }^{6}$ Hoeger Bement MK, Dicapo J, Rasiarmos R, Hunter SK. Dose response of isometric contractions on pain perception in healthy adults. Med Sci Sports Exerc. 2008;40(11):1880-1889.

${ }^{7}$ Yarnitsky D. Conditioned pain modulation (the diffuse noxious inhibitory control-like effect): its relevance for acute and chronic pain states. Curr Opin Anaesthesiol. 2010;23(5):611-615.

${ }^{8}$ van Wijk G, Veldhuijzen DS. Perspective on diffuse noxious inhibitory controls as a model of endogenous pain modulation in clinical pain syndromes. J Pain. 2010;11(5):408-419.

${ }^{9}$ Edwards RR, Ness TJ, Weigent DA, Fillingim RB. Individual differences in diffuse noxious inhibitory controls (DNIC): association with clinical variables. Pain. 2003;106(3):427-437.

${ }^{10}$ Lariviere M, Goffaux P, Marchand S, Julien N. Changes in pain perception and descending inhibitory controls start at middle age in healthy adults. Clin J Pain. 2007;23(6):506-510.

${ }^{11}$ Lemley KJ, Hunter SK, Bement MK. Conditioned pain modulation predicts exercise-induced hypoalgesia in healthy adults. Med Sci Sports Exerc. 2015;47(1):176-184.

${ }^{12}$ Stolzman S, Lemley K, Hoffmeister K, Coate M, Drendel A, Hoeger Bement M. Conditioned pain modulation and exercise-induced hypoalgesia in adolescents. Pediatr Phys Ther.

2014;26(1):154-155.

Pediatric Physical Therapy, Vol. 28, No. 4 (Winter 2016): pg. 470-473. DOI. This article is @ Lippincott Williams \& Wilkins, Inc. and permission has been granted for this version to appear in e-Publications@Marquette. Lippincott Williams \& Wilkins, Inc. does not grant permission for this article to be further copied/distributed or hosted elsewhere without the express permission from Lippincott Williams \& Wilkins, Inc.. 
13Stolzman S, Hoeger Bement M. Lean mass predicts conditioned pain modulation in adolescents across weight status. Eur J Pain. 2016;20(6):967-976.

${ }^{14}$ Tsao JC, Seidman LC, Evans S, Lung KC, Zeltzer LK, Naliboff BD. Conditioned pain modulation in children and adolescents: effects of sex and age. J Pain. 2013;14(6):558-567.

15Vaegter HB, Handberg G, Jorgensen MN, Kinly A, Graven-Nielsen T. Aerobic exercise and cold pressor test induce hypoalgesia in active and inactive men and women. Pain Med. 2015;16(5):923-933.

${ }^{16}$ Serlin RC, Mendoza TR, Nakamura Y, Edwards KR, Cleeland CS. When is cancer pain mild, moderate or severe? Grading pain severity by its interference with function. Pain. 1995;61(2):277-284.

17Turner JA, Franklin G, Heagerty PJ, et al. The association between pain and disability. Pain. 2004;112(3):307-314.

18Price RC, Asenjo JF, Christou NV, Backman SB, Schweinhardt P. The role of excess subcutaneous fat in pain and sensory sensitivity in obesity. Eur J Pain. 2013;17(9):1316-1326.

${ }^{19}$ Ellingson LD, Koltyn KF, Kim JS, Cook DB. Does exercise induce hypoalgesia through conditioned pain modulation? Psychophysiology. 2014;51(3):267-276.

\section{Table 1}

TABLE 1 Participant Characteristics $(n=55)$

\begin{tabular}{|c|c|}
\hline & Mean \pm SD \\
\hline Sex (male) & 26 \\
\hline Age, y & $15.2 \pm 1.8$ \\
\hline BMI Z score & $0.98 \pm 0.93$ \\
\hline Lean mass-whole body, $\mathrm{kg}$ & $46.8 \pm 10.9$ \\
\hline Lean mass-left am, kg & $2.7 \pm 0.9$ \\
\hline Peak pain during maximal exercise (NRS $0-10$ ) & $4.3 \pm 2.9$ \\
\hline $\mathrm{CPM}_{\text {Naindel, }} \mathrm{kPa}$ & $84.6 \pm 85.2$ \\
\hline $\mathrm{CPM} \mathrm{kPa}$ & $77.4 \pm 100.1$ \\
\hline $\mathrm{CPM}_{\mathrm{O}} \mathrm{kPa}$ & $91.8 \pm 123.4$ \\
\hline $\mathrm{ElH}_{\mathrm{Au}} \mathrm{kPa}$ & $29.5 \pm 87.4$ \\
\hline $\mathrm{ElH}_{\mathrm{Nai}}, \mathrm{kPa}$ & $0.8 \pm 74.9$ \\
\hline $\mathrm{ElH}_{\mathrm{Det}} \mathrm{kPa}$ & $27.9 \pm 69.5$ \\
\hline $\mathrm{ElH}_{\text {Ouad, }} \mathrm{kPa}$ & $56.1 \pm 101.4$ \\
\hline $\mathrm{ElH}_{\text {Detouad, }} \mathrm{kPa}$ & $42.0 \pm 64.3$ \\
\hline
\end{tabular}

musde; $S D$, standard deviation. 
NOT THE PUBLISHED VERSION; this is the author's final, peer-reviewed manuscript. The published version may be accessed by following the link in the citation at the bottom of the page.

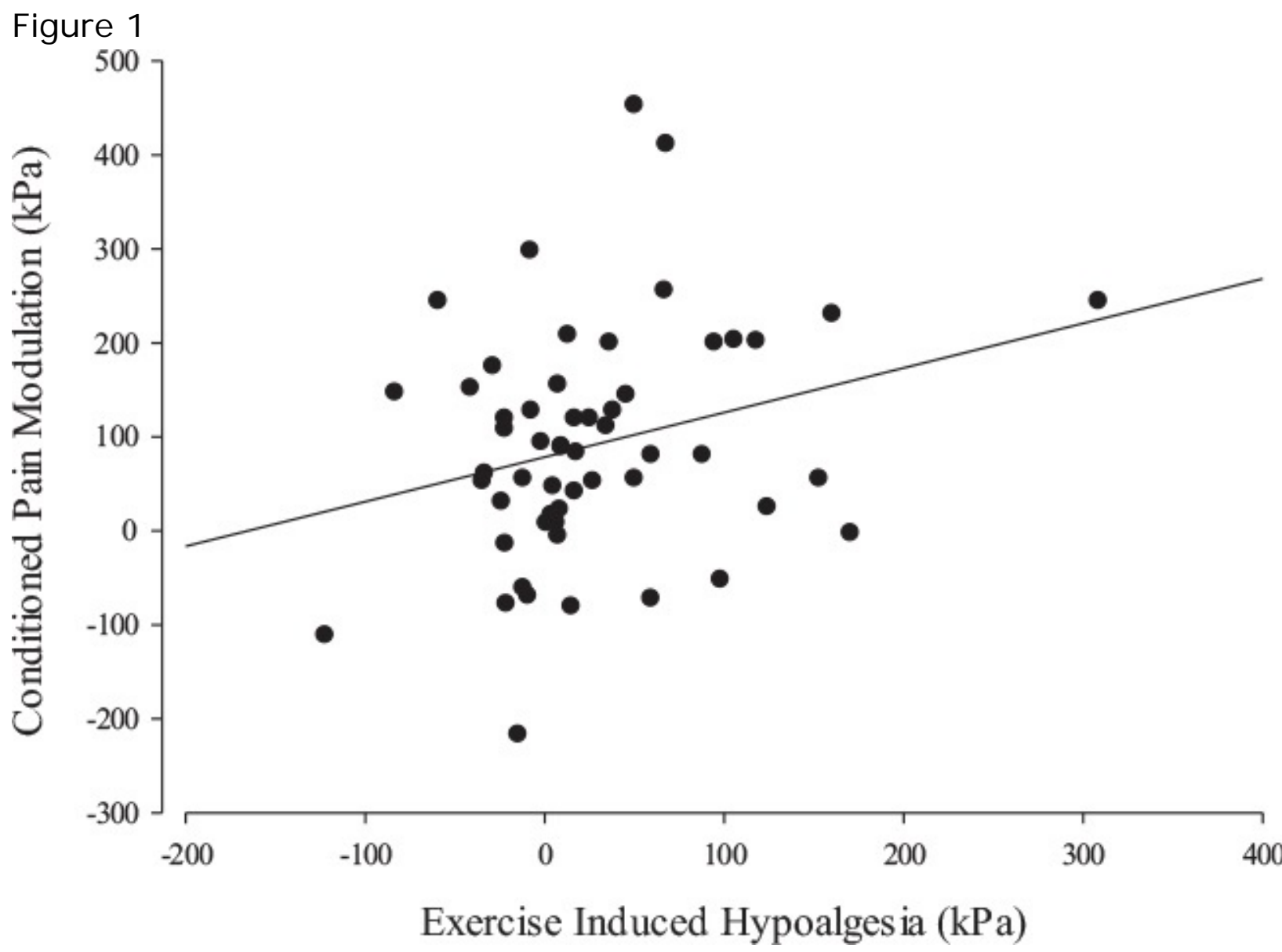

Pediatric Physical Therapy, Vol. 28, No. 4 (Winter 2016): pg. 470-473. DOI. This article is ( Lippincott Williams \& Wilkins, Inc. and permission has been granted for this version to appear in e-Publications@Marquette. Lippincott Williams \& Wilkins, Inc. does not grant permission for this article to be further copied/distributed or hosted elsewhere without the express permission from Lippincott Williams \& Wilkins, Inc.. 
NOT THE PUBLISHED VERSION; this is the author's final, peer-reviewed manuscript. The published version may be accessed by following the link in the citation at the bottom of the page.

TABLE 2 Relationship Between CPM and EIH at the Deltoid and Nail Bed Sites

\begin{tabular}{|c|c|c|c|}
\hline & $\mathrm{CPM}_{\text {NailDelt }}$ & $\mathrm{CPM}_{\text {Nail }}$ & $\mathrm{CPM}_{\text {Delt }}$ \\
\hline $\mathrm{ElH}_{\text {DetOuad }}$ & $r=0.20, P=.15$ & $r=0.07, P=.61$ & $r=0.21, P=.12$ \\
\hline $\mathrm{ElH}_{\text {Det }}$ & $r=0.23, P=.09$ & $r=0.06, P=.67$ & $r=0.27, P=.05$ \\
\hline $\mathrm{ElH}_{\text {Ouad }}$ & $r=0.09, P=.50$ & $r=0.05, P=.72$ & $r=0.09, P=.52$ \\
\hline
\end{tabular}

Abbreviations: CPM, œonditioned pain modulation; Delt, deltoid; EIH, exercise-induœed hypoalgesia; Nail, nail bed; Quad, quadriceps.

Pediatric Physical Therapy, Vol. 28, No. 4 (Winter 2016): pg. 470-473. DOI. This article is ( Lippincott Williams \& Wilkins, Inc. and permission has been granted for this version to appear in e-Publications@Marquette. Lippincott Williams \& Wilkins, Inc. does not grant permission for this article to be further copied/distributed or hosted elsewhere without the express permission from Lippincott Williams \& Wilkins, Inc.. 


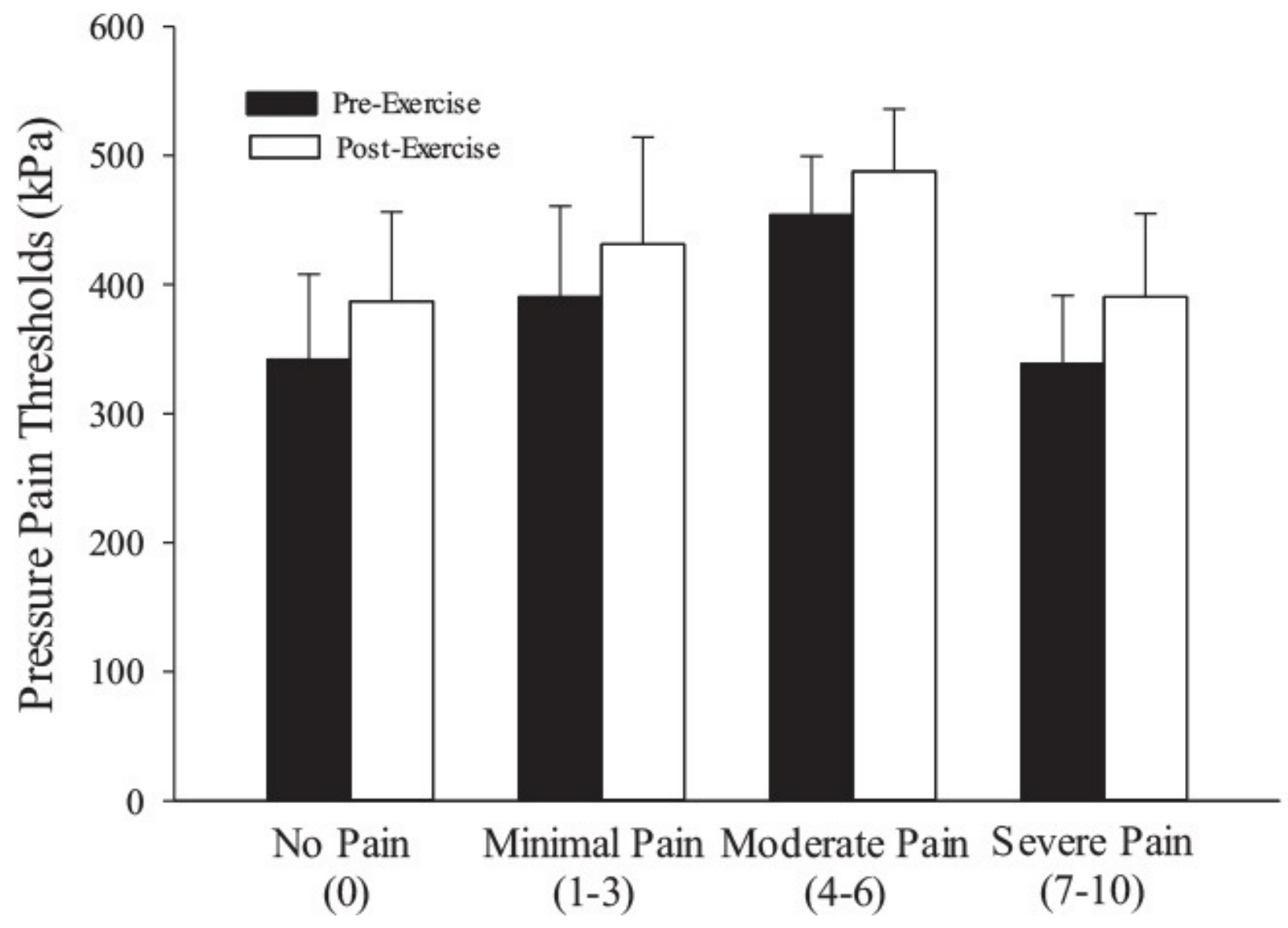

Pediatric Physical Therapy, Vol. 28, No. 4 (Winter 2016): pg. 470-473. DOI. This article is ( Lippincott Williams \& Wilkins, Inc. and permission has been granted for this version to appear in e-Publications@Marquette. Lippincott Williams \& Wilkins, Inc. does not grant permission for this article to be further copied/distributed or hosted elsewhere without the express permission from Lippincott Williams \& Wilkins, Inc.. 